\title{
CARCINOSARCOMA VESICAL: ANÁLISIS DE NUESTRA SERIE Y REVISIÓN DE LA LITERATURA
}

\author{
E. ARGÜElleS SALIDO, P. TRAVADO SORIA*, Mํㅗ. PÉREZ ESPEJO, J. RODRÍGUEZ \\ CORCHERO, R.A. MEDINA LÓPEZ, J.Mª PENA OUTEIRIÑO
}

Servicio de Urología. *Servicio de Anatomía Patológica. Hospital Universitario Virgen del Rocío. Sevilla.

Actas Urol Esp. 28 (3): 262-268, 2004

\section{RESUMEN}

CARCINOSARCOMA VESICAL: ANÁLISIS DE NUESTRA SERIE Y REVISIÓN DE LA LITERATURA.

El carcinosarcoma vesical es una neoplasia de muy baja frecuencia de aparición. Por ello su historia natural y las formas de tratamiento no están claramente determinadas. Suelen ser tumores rápido crecimiento, invasivos y con tendencia a la recurrencia.

Presentamos la revisión de los cuatro casos que hemos tenido ocasión de diagnosticar y tratar entre 1995 y 2002. Analizamos los aspectos epidemiológicos, sintomatología presentada, hallazgos histológicos, métodos diagnósticos, tratamiento y supervivencia, revisando además la literatura al respecto.

PALABRAS CLAVE: Carcinosarcoma. Tumores vesicales. Vejiga Urinaria. Tumor mesodérmico mixto.

\section{ABSTRACT \\ CARCINOSARCOMA OF THE BLADDER: REPORT OF OUR CASES AND REVIEW OF THE LITERATURE.}

Carcinosarcomas of the bladder are rare. As a result, the natural history of them and the best methods of treatment remain uncertain. These tumors tend to be rapidly growing, invasive and recur locally.

We reviewed our experience with four patients presenting between 1995 and 2002. Epidemiology findings, clinical aspects, histological features, diagnostic methods, treatment and survival are discussed in relation to literature.

KEY WORDS: Carcinosarcoma. Bladder tumors. Urinary bladder. Mixed mesodermal tumour.

$\mathrm{E}_{\mathrm{s}}$ carcinosarcoma (CS) vesical es una neoplasia extremadamente infrecuente, dado que representan menos del $0 \_5 \%$ de las neoplasias vesicales $^{1}$.

Son tumores constituidos por dos componentes; uno formado por epitelio de características malignas (carcinoma) y otro de características sarcomatosas procedente de derivados mesenquimatosos.
Ha sido descrito fundamentalmente en el tracto genital femenino, sobre todo en el útero, aunque también se han desarrollado en otras localizaciones como gastrointestinal, sistema hepatobiliar, cuello y cabeza, sistema respiratorio ${ }^{2}$...

En los últimos 50 años han sido publicados en la literatura médica en lengua inglesa ${ }^{3}$ unos 12 casos de CS de riñón ${ }^{4}, 10$ de uréter $^{5}$ (en alguno de los casos provocando obstrucción del mismo) ${ }^{6}$, 
83 de vejiga, 20 de próstata ${ }^{7}$ y 2 de pene. Aparecieron comunicados en su mayoría como casos clínicos individuales o pequeñas series. En lo que al CS vesical concierne, destacan la serie de LÓPEZ BELTRÁN et al. ${ }^{8}$ con 15 casos y la revisión de 55 casos realizada en 1997 por PERRET et al, en la que se aportaban además 8 nuevos casos.

El objetivo de este trabajo es analizar nuestra serie de CS vesicales de los últimos 7 años, además de hacer una revisión de la literatura hasta el momento.

\section{MATERIAL Y MÉTODO}

Hemos realizado un estudio retrospectivo de los casos de CS vesical que han sido diagnosticados y tratados en el Servicio de Urología del Hospital Universitario Virgen del Rocío desde 1995 a 2002.

Estudiamos las variables edad, sexo, antecedentes personales, clínica de presentación, diagnóstico, resultado del estudio anatomopatológico, tratamiento y supervivencia en los cuatro pacientes hallados, tres hombres y una mujer, de edades comprendidas entre los 49 y los 80 años (edad media de 65 años).

\section{RESULTADOS}

Los casos estudiados, con una mayor incidencia en varones $(3 / 1)$, se muestran resumidos en la Tabla I.

Como antecedentes personales de interés destaca el tabaquismo, presente en el $75 \%$ de los pacientes. Dos de ellos poseían además antecedentes de resección transuretral (RTU) de carcinomas vesicales de células transicionales (CCT). Otros antecedentes personales fueron diabetes mellitus insulín dependiente, tuberculosis pulmonar, cardiopatía isquémica con expresión de infarto agudo de miocardio, litiasis renal, herniorrafia inguinal e intervención por cataratas. Como antecedentes familiares destacamos que en uno de los pacientes encontramos dos fallecimientos por neoplasia vesical.

La clínica de debut más frecuente (3 de los 4 casos) fue la hematuria total con coágulos. El caso restante presentó inicialmente un síndrome constitucional acompañado de sintomas miccionales irritativos. Como síntoma acompañante más frecuente encontramos el dolor suprapúbico.

En todos los pacientes el diagnóstico de neoformación vesical se realizó mediante ecografía. Este estudio se completó además en dos de los

TABLA I

\begin{tabular}{|c|c|c|c|c|}
\hline & CASO 1 & CASO 2 & CASO 3 & CASO 4 \\
\hline Edad & 49 & 53 & 78 & 80 \\
\hline Sexo & Hombre & Mujer & Hombre & Hombre \\
\hline Antecedentes & Dmid. & $\begin{array}{l}\text { Fumadora } \\
\text { A. Familiares } \\
\text { (padre y tío) }\end{array}$ & $\begin{array}{l}\text { Fumador } \\
\text { RTU dos años antes: } \\
\text { Ca papilar } \\
\text { urotelial grado II }\end{array}$ & $\begin{array}{l}\text { Fumador, TBC } \\
\text { residual, IAM. } \\
\text { RTU: Ca papilar } \\
\text { urotelial grado I }\end{array}$ \\
\hline $\begin{array}{l}\text { Clínica de } \\
\text { debut }\end{array}$ & $\begin{array}{l}\text { Hematuria } \\
\text { total con } \\
\text { coágulos, } \\
\text { disuria, } \\
\text { polaquiuria, } \\
\text { dolor } \\
\text { suprapúbico }\end{array}$ & $\begin{array}{l}\text { S. Irritativos } \\
\text { Dolor } \\
\text { suprapúbico. } \\
\text { Astenia y } \\
\text { pérdida de peso }\end{array}$ & $\begin{array}{l}\text { Hematuria con } \\
\text { coágulos }\end{array}$ & $\begin{array}{l}\text { Hematuria con } \\
\text { coágulos }\end{array}$ \\
\hline Tratamiento & $\begin{array}{l}\text { RTU } \\
\text { Cistectomía } \\
\text { (Bricker) }\end{array}$ & $\begin{array}{l}\text { RTU } \\
\text { Cistectomía } \\
\text { parcial }\end{array}$ & $\begin{array}{l}\text { RTU de } \\
\text { repetición }\end{array}$ & RTU paliativa \\
\hline Supervivencia & 6 meses & $\begin{array}{l}\text { Vive } \\
\text { (3,5 años) }\end{array}$ & 1,5 años & $\begin{array}{l}\text { Vive } \\
\text { (10 meses) }\end{array}$ \\
\hline
\end{tabular}


casos con urografía intravenosa, encontrándose en ambas un defecto de replección vesical concordante con los hallazgos ecográficos. (Fig. 1). La tomografía axial computerizada de los pacientes que iban a ser cistectomizados no mostró extensión tumoral extravesical. No se realizó en los pacientes restantes puesto que dada la edad que tenían (78 y 80 años) no eran candidatos a cirugía radical.

En el $100 \%$ de los pacientes el procedimiento terapéutico inicial fue la RTU de la neoformación. En el $50 \%$ de ellos éste fue el único tratamiento empleado. De los pacientes restantes uno se sometió a una cistectomía radical y ureteroileostomía cutánea tipo Bricker, y otro a una cistectomía parcial.

El 50\% de los pacientes fallecieron dentro de los primeros 18 meses tras la cirugía debido a la patología tumoral. Uno de ellos lo hizo 6 meses después de la RTU, y el otro 18 meses después de

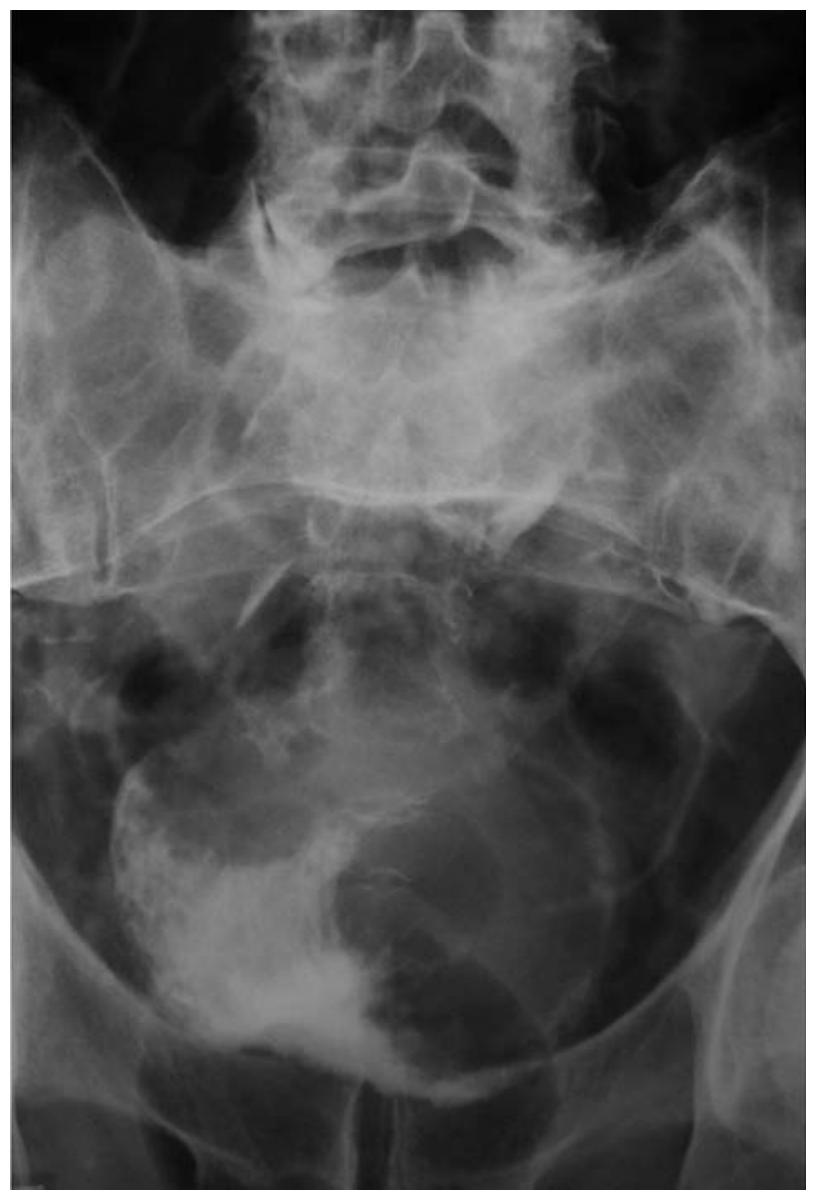

FIGURA 1. UIV del caso no ${ }^{-4}$, en la que se observa el defecto de replección vesical producido por la neoplasia. la cistectomía radical. Los otros dos pacientes continúan vivos. A uno se le práctico RTU, habiéndose revisado por última vez 10 meses después del procedimiento quirúrgico. La paciente sometida a cistectomía parcial presenta hasta el momento una supervivencia de 3,5 años.

Los resultados anatomopatológicos han sido resumidos en la Tabla II.

En el 75\% de los casos el componente epitelial del CS fue mixto: dos de los tumores tenían componente transicional, uno acompañado de elementos glandulares y otro con zonas escamosas (Fig. 2). En el otro individuo se observaban elementos glandulares y escamosos entremezclados. En el paciente con único componente epitelial éste resultó carcinoma transicional.

Respecto a los componentes mesenquimatosos encontramos elementos rabdo y osteosarcomatosos en un caso, elementos fusocelulares en dos de los pacientes (uno con cierta diferenciación rabdoide), y componentes osteo y condrosarcomatosos en el restante. (Fig. 3).

Todos los casos presentaron inmunoreactividad citoplasmática para citokeratina y vimentina (Fig. 4). Uno presentaba además inmunoreactividad para la desmina y otro para actina de músculo liso (AML).

\section{DISCUSIÓN}

Aunque se atribuye a Shattock la descripción de esta patología, en 1887, fue Wirchow quien propuso el término de carcinosarcoma ${ }^{9}$. La Organización Mundial de la Salud los define como tumores compuestos por epitelio maligno y elementos mesenquimatosos en íntima unión.

Esta neoformación afecta principalmente a varones (1,7-4 hombres: 1 mujeres) $)^{2,8}$ en la edad media de la vida, aunque con un rango de edad amplio, desde los 21 a los 91 años, y una edad media de 66,4, lo que coincide con los tumores vesicales habituales. Esto coincide con la media de edad encontrada en nuestra serie.

Desde su descripción la histogénesis exacta de estas neoplasias ha permanecido enigmática e incluso actualmente continua siendo uno de los temas controvertidos en patologia tumoral ${ }^{10}$

Macroscópicamente estos tumores aparecen como masas polipoideas exofiticas con áreas de necrosis y ulceración, que crecen en la luz de vísceras huecas (Fig. 5). 


\section{TABLA II}

\begin{tabular}{|c|c|c|c|}
\hline CASO 1 & CASO 2 & CASO 3 & CASO 4 \\
\hline $\begin{array}{l}\text { Epitelio transicional y } \\
\text { Escamoso: citokeratina } \\
\text { (Ck) A622 y A575+ }\end{array}$ & $\begin{array}{l}\text { Epitelio transicional: } \\
\text { Ck z622+ y } 8,18+\end{array}$ & $\begin{array}{l}\text { Epitelio escamoso } \\
\text { y glandular: Ck z6 } \\
22+\text { y } 8,18+\end{array}$ & $\begin{array}{l}\text { Epitelio transicional } \\
\text { y escamoso. Ck } 7+\end{array}$ \\
\hline $\begin{array}{l}\text { Mesénquima: Rabdo y } \\
\text { osteosarcoma. } \\
\text { Vimentina + Desmina y } \\
\text { mioglobina - }\end{array}$ & $\begin{array}{l}\text { Mesénquima: células } \\
\text { (cels) fusiformes } \\
\text { indiferenciadas. } \\
\text { Vimentina + Desmina- } \\
\text { Actina muscular lisa } \\
\text { (AML)+ }\end{array}$ & $\begin{array}{l}\text { Mesénquima: } \\
\text { fusocelular } \\
\text { Rabdoide: } \\
\text { Desmina + en cels. } \\
\text { rabdoides } \\
\text { Vimentina + en } \\
\text { cels. fusiformes }\end{array}$ & $\begin{array}{l}\text { Mesénquima: cóndor } \\
\text { y osteosarcoma } \\
\text { Vimentina }+\end{array}$ \\
\hline
\end{tabular}

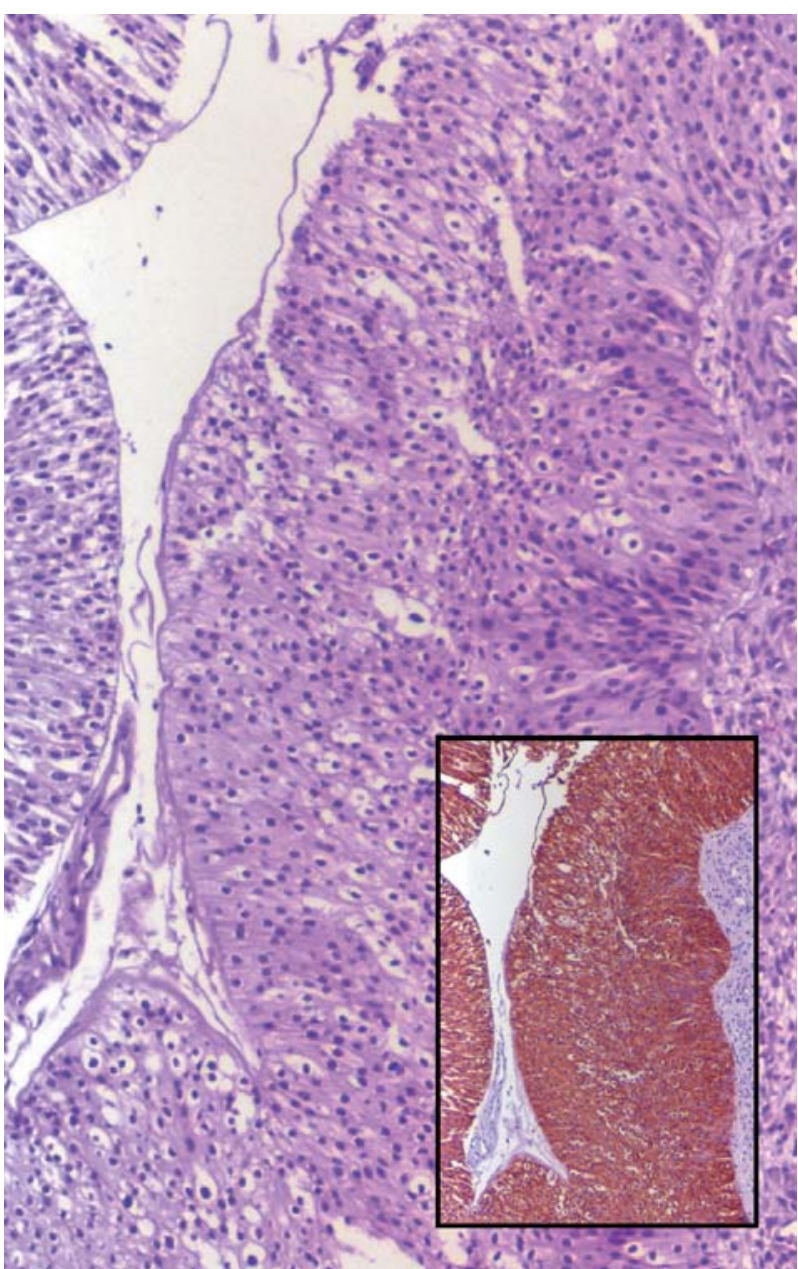

FIGURA 2. Carcinoma de células transicionales (hematoxilina eosina). En el recuadro inferior observamos zonas positivas para antigeno de membrana epitelial (EMA), con un limite neto a la derecha de la imagen que ya no se tiñe y que corresponde a la zona sarcomatosa.

Hay acuerdo en lo que al componente epitelial se refiere. Así el más frecuente es el carcinoma transicional de alto grado (80\%), seguido por el de células escamosas (32\%), los de diferenciación adenomatoide (26\%) y finalmente el carcinoma de células pequeñas (5\%). En el 33\% de las ocasiones se presentan dos o más componentes epiteliales. En nuestra serie sin embargo lo más fre-

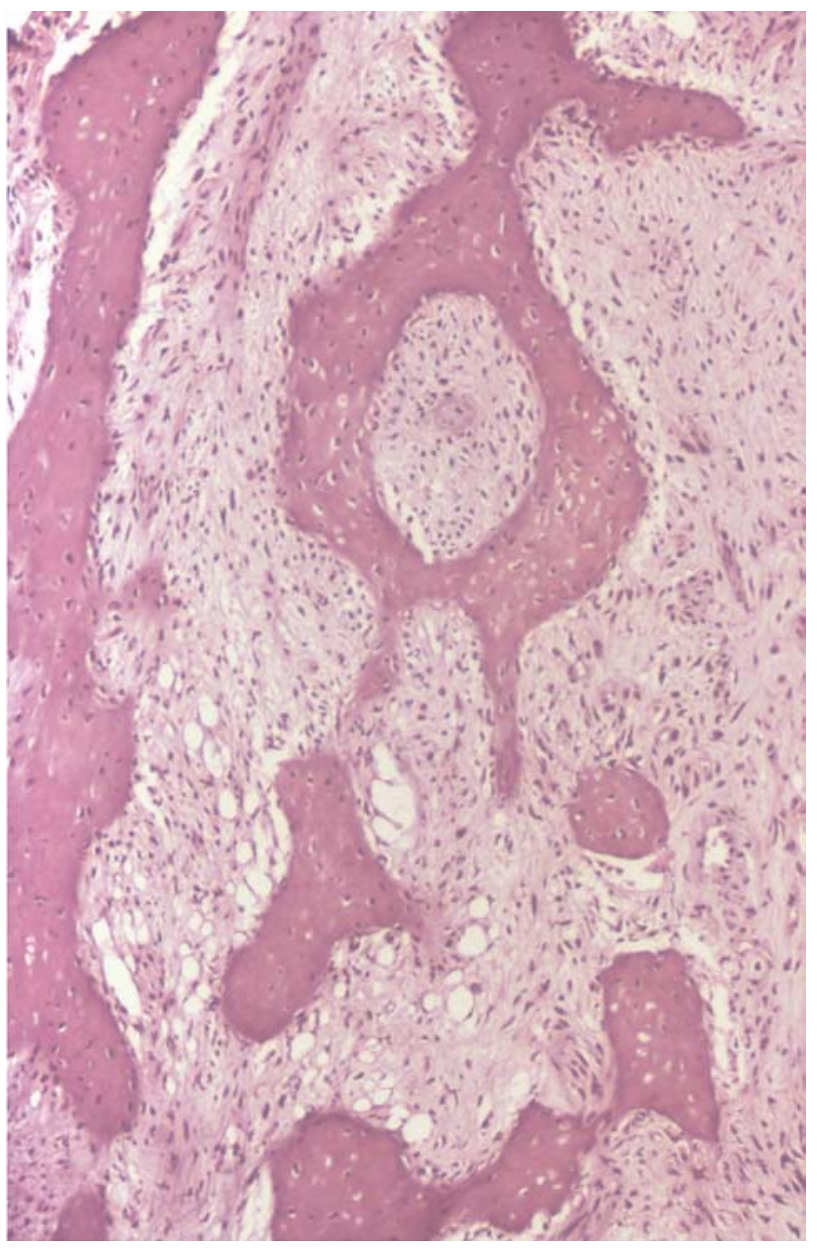

FIGURA 3. Tinción con hematoxilina-eosina en la que se aprecian zonas de matriz ósea bastante madura quedando pocos islotes celulares. 


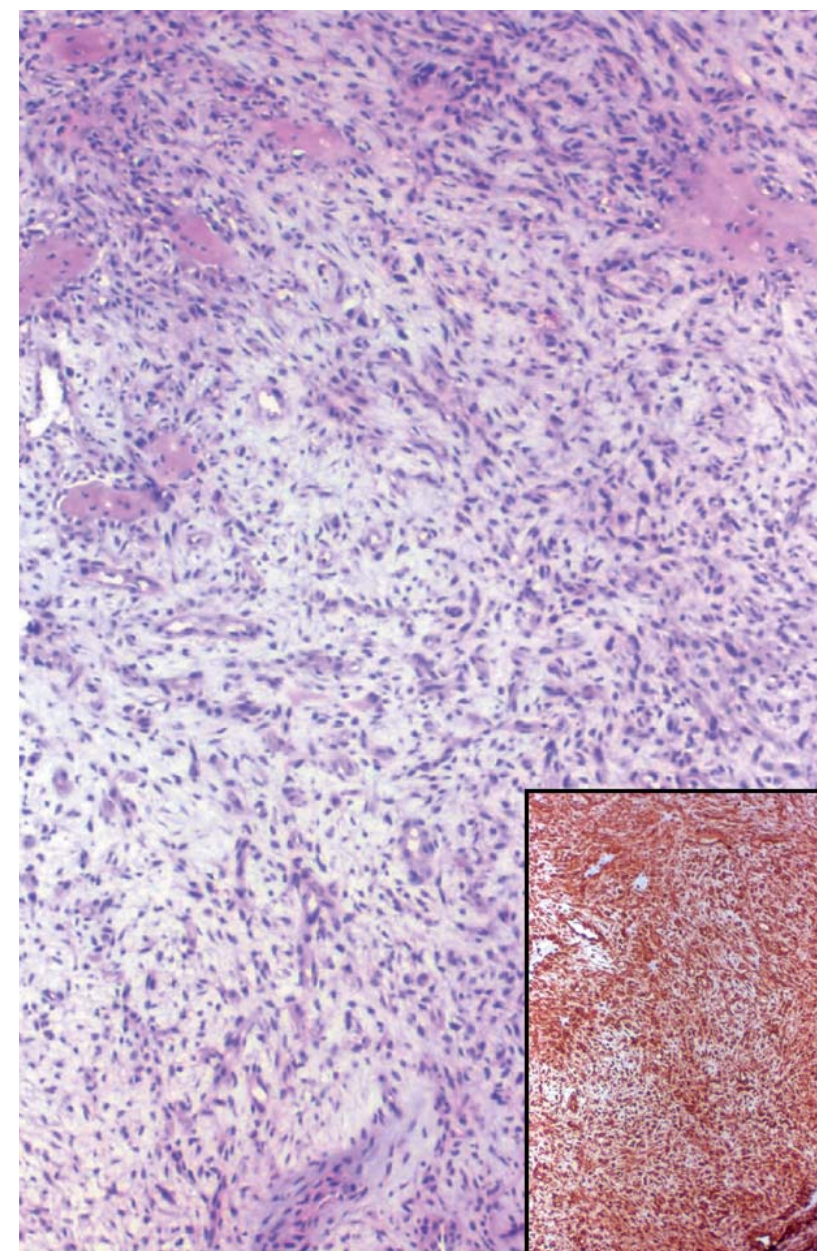

FIGURA 4. Tinción con hematoxilina-eosina de la zona de sarcoma indiferenciado: Células fusiformes con núcleo elongado, en forma de bala. En el recuadro inferior lo observamos teñido con vimentina, lo que se corresponde con tejido de procedencia mesenquimatosa.

cuente ha resultado ser un componente epitelial mixto (75\% de las ocasiones), siendo el carcinoma transicional efectivamente el más frecuente.

En cuanto al componente mesodérmico o conjuntivo, existen diferencias en la literatura en cuanto a su distribución por frecuencia, siendo para algunos la más frecuente la diferenciación ósea ${ }^{10}$ con un $37 \%$, y para otros la cartilaginosa (20-47\%) siendo menos habitual los componentes rabdomiomatosos, fusiformes indiferenciados, etc. ${ }^{1,2}$. En nuestra muestra el $50 \%$ presentaba elementos osteosarcomatosos.

Como se mencionó antes, la histogénesis del CS continúa teniendo puntos oscuros, habiendo existido desacuerdo respecto a su nomenclatura. De esta forma encontramos las teorías de:

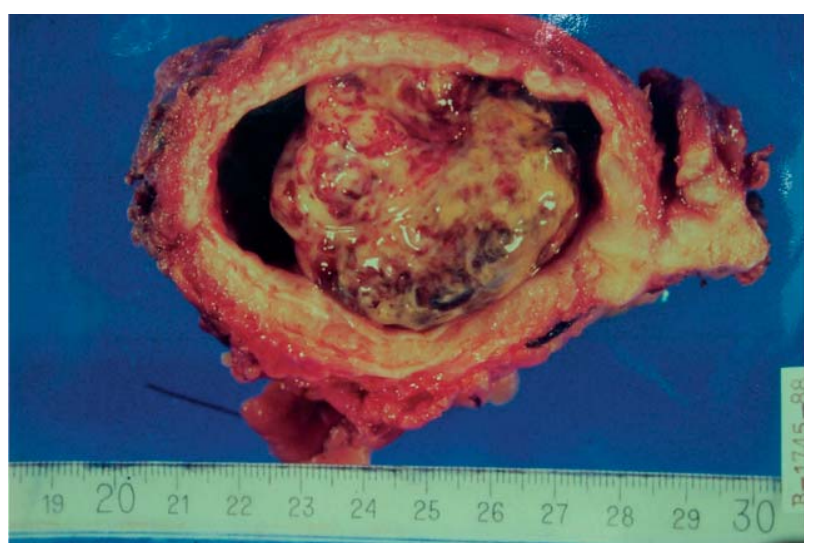

FIGURA 5. Aspecto macroscópico de la pieza de cistectomía radical, con una masa polipoidea exofitica con áreas de necrosis y ulceración, de crecimiento endovesical.

- Colisión tumoral: basado en la presencia de dos tumores malignos independientes que se invaden entre $\mathrm{si}^{11,12}$.

- Tumores originados en una misma célula indiferenciada (stem cell): apoyado en la posibilidad de hallar inmunoreactividad para marcadores epiteliales como la citokeratina o antígeno de membrana epitelial (EMA) en las áreas mesenquimatosas, además de hallazgos ultraestructurales como desmosomas o tonofilamentos en las áreas sarcomatosas ${ }^{10}$.

- Metaplasia de elementos epiteliales malignos (carcinosarcoma) a elementos sarcomatoides en el estroma al encontrar tinción para queratina (AE1/AE3) no sólo en el componente epitelial sino también en el mesenquimatoso ${ }^{13}$.

Se han sugerido principalmente tres factores etiológicos relacionados con esta patología:

- Radiación ${ }^{2}$ : está demostrada la asociación entre antecedentes de radioterapia y aparición del CS uterino. Esta relación no está confirmada en el CS vesical, aunque parece probable dada la existencia de tratamiento radioterápico previo en algunos de estos pacientes ${ }^{14}$. Ninguno de los pacientes diagnosticados en nuestro hospital habian recibido radioterapia previamente al diagnóstico de CS.

- Tabaco: al igual que en los carcinomas de urotelio convencionales, parece ser también un factor etiológico en los CS. Por ello se ha estimado la posibilidad de que el CS y el CCT compartan factores favorecedores o inductores carcinogénicos, al haberse publicado casos de coexisten- 
cia de ambos en una misma vejiga ${ }^{10,15}$. En nuestros casos se encuentra una amplia frecuencia de este antecedente personal (75\%).

- La ciclofosfamida administrada durante un periodo prolongado es también considerada como un factor etiológico ${ }^{2,16}$. Ninguno de nuestros pacientes había recibido este quimioterápico previamente al diagnóstico de CS.

La clínica del CS en localización vesical no difiere de la presentada por el habitual CCT: hematuria total con coágulos como sintomatología más frecuente, seguida de disuria e infección urinaria ${ }^{1,9,12,17}$. Estos datos coinciden prácticamente con los de nuestra casuística, aunque el debut como sindrome constitucional que vemos en uno de los pacientes no es frecuente.

El diagnóstico se basa fundamentalmente en el examen convencional con hematoxilina-eosina, y en el estudio inmunohistoquímico. En casos necesarios se puede recurrir a la microscopía electrónica. En su análisis inmunohistoquímico las células epiteliales deben expresar citoqueratinas, que son filamentos proteicos característicos de células epiteliales, mientras que el componente mesenquimatoso debe expresar vimentina, desmina, HHF-35 (rabdomiosarcoma), SMA o S100 (cartílago).

El diagnóstico por las pruebas de imagen no nos aporta rasgos diferenciales entre el CCT típico y el CS.

El diagnóstico diferencial debe realizarse con sarcomas primarios, carcinomas primarios con metaplasia estromal, carcinomas con estroma pseudosarcomatoso, sarcomas asociados a hiperplasia pseudosarcomatosa, sarcomas asociados a hiperplasia pseudoepiteliomatosa y terato$\operatorname{mas}^{2,10,18}$.

La diferencia fundamental entre el CCT y el CS es su pronóstico, infausto en la gran mayoría de las ocasiones en éste último, con $20 \%$ de supervivencia a los 5 años $^{1}$. De hecho, ya en el momento del diagnóstico la mayoría de los casos presentan estado avanzado ( $83 \%$ alguna serie) ${ }^{2}$. No obstante se han descrito casos de supervivencia larga, incluso mayor de doce años, que no invalidan el diagnostico ${ }^{16}$. Encontramos un ejemplo similar en nuestra paciente sometida a cistectomía parcial, con 3,5 años de supervivencia hasta el momento.
La diseminación es mayoritariamente locorregional por vía linfática, y en menos ocasiones por vía hematógena ${ }^{18}$.

Parece que la composición histológica del tumor no influye en el pronóstico, siendo el estadiaje el factor que más orienta ${ }^{8}$.

En general, siempre que sea posible, el tratamiento quirúrgico debe ser agresivo. Ni la radioterapia ni la quimioterapia sistémica parecen ser eficaces $^{2,8}$.

No obstante la protocolización del tratamiento no es fácil, debido tanto a la escasez de casos como a los pobres resultados independientemente de la terapia seguida. En la serie de BASCHINDSKY que revisa 78 casos bien documentados, en los que al $79 \%$ de los pacientes sólo se le realizó tratamiento quirúrgico, al 9\% se les añadió radioterapia, al 3\% se les añadió quimioterapia, y en un $3 \%$ ambas. Un $6 \%$ no recibió tratamiento.

Nuestros pacientes fueron tratados todos de forma únicamente quirúrgica. Dos de ellos fueron sometidos sólo a RTU de la neoformación vesical ante lo avanzado de su edad. En el caso de la cistectomía parcial, la paciente se negó a que se le practicara una cirugía más agresiva.

\section{CONCLUSIONES}

El carcinosarcoma es una neoplasia de muy escasa incidencia urológica.

$\mathrm{Su}$ agresiva evolución junto a su compleja anatomía patológica son los parámetros que lo distinguen del CCT, puesto que ni su clínica ni las pruebas de imagen son específicas.

Debido a su escasa frecuencia de presentación no existe un tratamiento estandarizado.

\section{REFERENCIAS}

1. SÁNCHEZ BERNAL C, BAEZ PEREA JM, BACHILLER BURGOS J, SOTO DELGADO M, JUÁREZ SOTO A, BELTRÁN AGUILAR V: Carcinosarcoma vesical: aportación de un caso con estudio inmunohistológico. Actas Urol Esp 1998; 22 (7): 557-560.

2. PERRET L, CHAUBERT P, HESSLER D, GUILLOU L: Primary heterologous carcinosarcoma (metaplastic carcinoma) of the urinary bladder. A clinicopathologic, immunohistochemical and ultraestructural analysis of eight cases and review of the literature. Cancer 1998; 82 (8): 1.535-1.549.

3. LEMA GRILLE J, BLANCO PARRA M, SUÁREZ PENAARANDA JM, DURANA TONDER C: Carcinosarcoma de pelvis renal: aportación de un caso y revisión de la literatura. Actas Urol Esp 2002; 26 (7): 509-512. 
4. DIMITRIOV RJ, GATTUSO P, COOGAN CL: Carcinosarcoma of the renal pelvis. Urology 2000; 56 (3): 508.

5. FLEMING S: Carcinosarcoma (mixed mesodermal tumor) of the ureter. $J$ Urol. 1987; 138: 1.2341.235 .

6. BYARD RW, ADELINE BELL ME, ALKAN MK: Primary carcinosarcoma: a rare cause of unilateral ureteral obstruction. J Urol 1987; 137: 732-733.

7. FUKAWA T, NUMATA $K$, YAMANAKA $M$ et al: Prostatic carcinosarcoma: a case report and review of the literature. Int $J$ Urol 2003; 10 (2): 108-213.

8. LÓPEZ-BELTRÁN A, PACELLI A, ROTHENBERG HJ, WOLLAN PC, ZINCKE H, BLUTE ML, BOSTWICK DG: Carcinosarcoma and sarcomatoid carcinoma of the bladder. Clinicopathological study of 41 cases. J Urol. 1998; 159 (5): 1.497-1.503.

9. PENA OUTEIRIÑO JM, LEÓN DUEÑAS E, ROMERO GIL JR, LEAL LÓPEZ A: Carcinosarcoma vesical. Caso clínico. Actas Urol Esp. 1995; 19 (3): 234 237.

10. BASCHINDKY DY, CHEN JH, VADMAL MS, LUCAS JG, BAHNSON RR, NIEMAN TH: Carcinosarcoma of the urinary bladder. An agresive tumor with diverse histogenesis. A clinicopathologic stydy of 4 cases and review of the literature. Arch Pathol Lab Med. 2000; 124 (8): 1.172-1.178.

11. PATTERSON TH AND DALE GA: Carcinosarcoma of the bladder: case report and review of the literature. J Urol 1976; 115: 753-755.

12. FIGUEIREDO L, NOGUEIRA MARCH JL, OJEA A, JAMARDO D, MATA J, CUNQUEIRO R, PÉREZ VILLANUEVA J: Carcinosarcoma vesical. A propósito de dos casos. Arch Esp Urol 1982; 35 (3): 179-182.
13. LAHOTI C, SCHINELLA R, RANGWALA AF, LEE M, MIZRACHI H: Carcinosarcoma of urinary bladder: report of 5 cases with immunohistologic study. Urology 1994; 43 (3): 389-393.

14. OLIVIER C, LAO AH, MONLLORH, CARBALLIDO J: Carcinosarcoma vesical con diferenciación osteosarcomatosa. Actas Urol Esp. 1991; 15 (4): 375380.

15. GONZÁLEZ-CARRERO J, NOGUERIRA MARCH JL, OJEA A, FIGUEIREDO D, JAMARDO D, GUATE JL, CUNQUEIRO R: Carcinosarcoma y carcinoma papilar de células transicionales coexistentes en la misma vejiga. Actas Urol Esp. 1990; 14: 286-288.

16. SIGAL SH, TOMASZEWSKI JE, BROOKS JJ, WEIN A, LIVOLSI VA: Carcinosarcoma of bladder following long-term cyclophosphamide therapy. Arch Pathol Lab Med. 1991; 115: 1.049-1.051.

17. GARMENDIA LARREA JC, RODRÍGUEZ ANDRÉS JA, CAMPA BORTOLO J, LOBO MORÁN C, LÓPEZ GARCÍA JA, AROCENA LANZ F: Carcinosarcoma con diferenciación condromatoide de vejiga. Arch Esp Urol. 1991; 44 (7): 861-862.

18. YOUNG RH: Carcinosarcoma of the urinary bladder. Cancer 1987; 59: 1.333-1.339.

Dr. E. Argüelles Salido

C/ Milano Plomizo no 4 , portal 4, $1^{\text {a }}$ B 41020 Sevilla

e-mail: earguelles@telefonica.net

(Trabajo recibido el 17 septiembre 2003) 